\title{
Correction to: Saroclazines A-C, thio-diketopiperazines from mangrove-derived fungi Sarocladium kiliense HDN11-84
}

\author{
Feng $\mathrm{Li}^{1} \cdot$ Wenqiang $\mathrm{Guo}^{1} \cdot \mathrm{Li} \mathrm{Wu}^{1} \cdot$ Tianjiao $\mathrm{Zhu}^{1} \cdot \mathrm{Qianqun}^{\mathrm{Gu}} \mathrm{u}^{1}$. \\ Dehai $\mathrm{Li}^{1,2} \cdot$ Qian Che $^{1}$ (D)
}

Published online: 6 February 2019

(C) The Pharmaceutical Society of Korea 2019

\section{Correction to: Arch. Pharm. Res. (2018) 41:30-34 \\ https://doi.org/10.1007/s12272-017-0961-7}

The author would like to include conflict of interest statement of the online published article. The correct conflict of interest statement should read as:
Conflict of interest The authors declare no conflict of interest.

Feng Li and Wenqiang Guo have contributed equally to this work.

The original article can be found online at https://doi.org/10.1007/s12272-017-0961-7.

Qian Che

cheqian064@ouc.edu.cn

Tianjiao Zhu

zhutj@ouc.edu.cn

Dehai Li

dehaili@ouc.edu.cn

1 Key Laboratory of Marine Drugs, Chinese Ministry of Education, School of Medicine and Pharmacy, Ocean University of China, Qingdao 266003, People's Republic of China

2 Laboratory for Marine Drugs and Bioproducts of Qingdao National Laboratory for Marine Science and Technology, Qingdao 266237, People's Republic of China 